
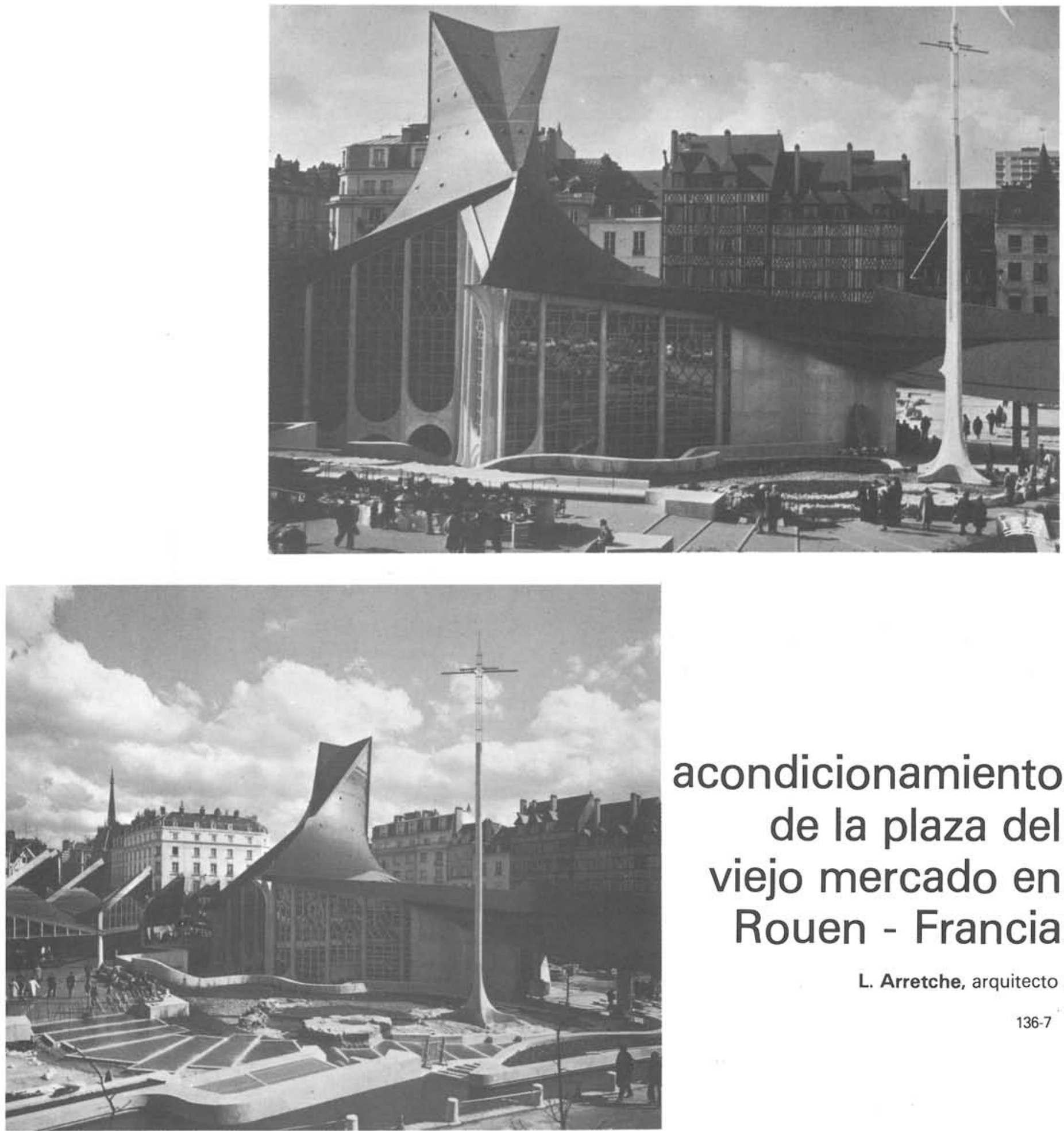

\title{
acondicionamiento \\ de la plaza del viejo mercado en Rouen - Francia
}

\section{sinopsis}

Con el acondicionamiento de la plaza del Viejo Mercado de Rouen se ha pretendido integrar en un conjunto armonioso tres elementos de tan distintas funciones como son:

- la iglesia,

- el mercado cubierto, y

- el monumento nacional a Juana de Arco.

Los diversos entramados se basan en la construcción naval tradicional y están constituidos por superficies regladas: paraboloides hiperbólicos hechos de madera.

Destacan en el conjunto las trece vidrieras del siglo XVI, salvadas milagrosamente de la destrucción de la iglesia de San Vicente.

\section{Arretche, arquitecto}

$136-7$ 


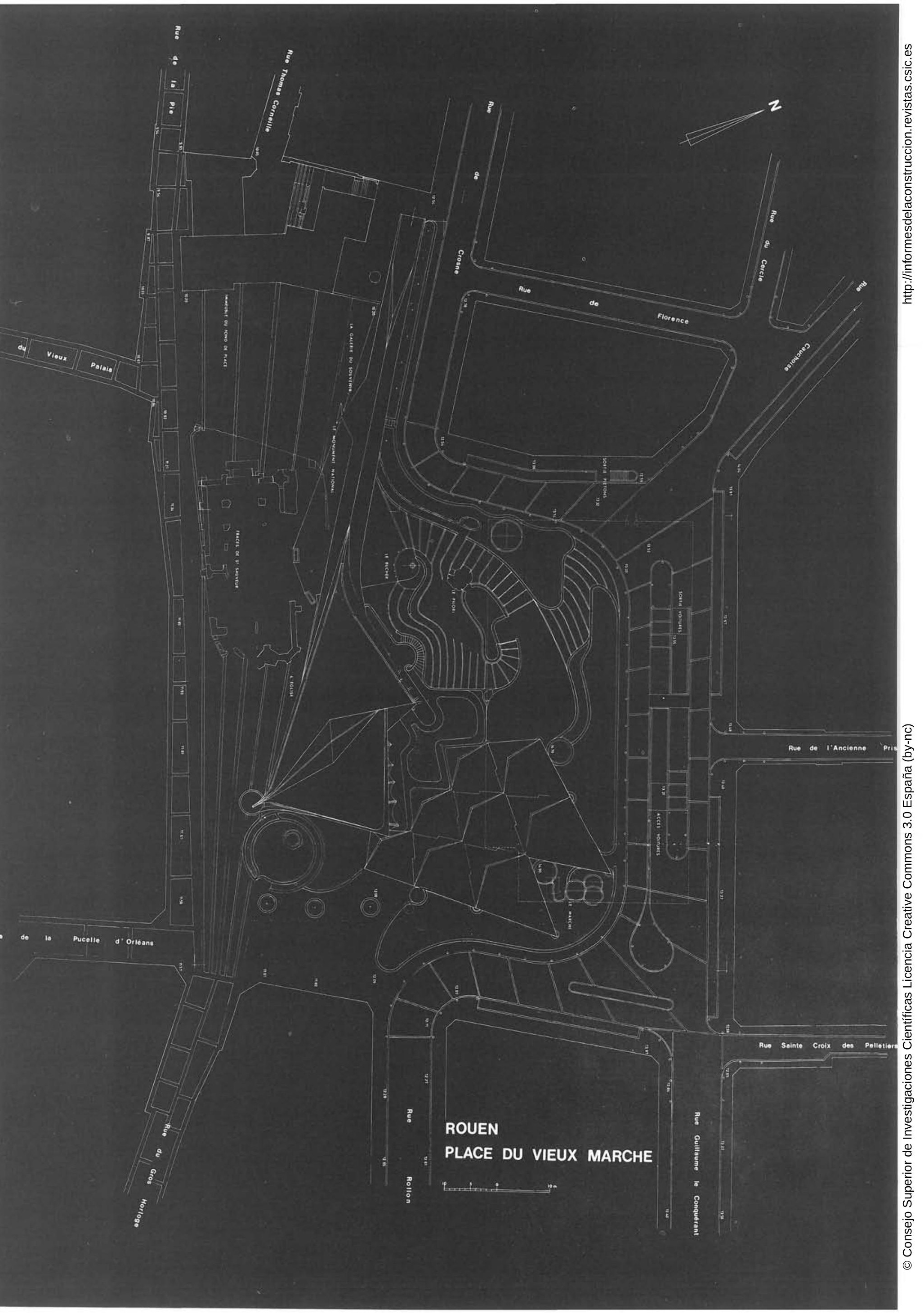




\section{vistas de la estructura}

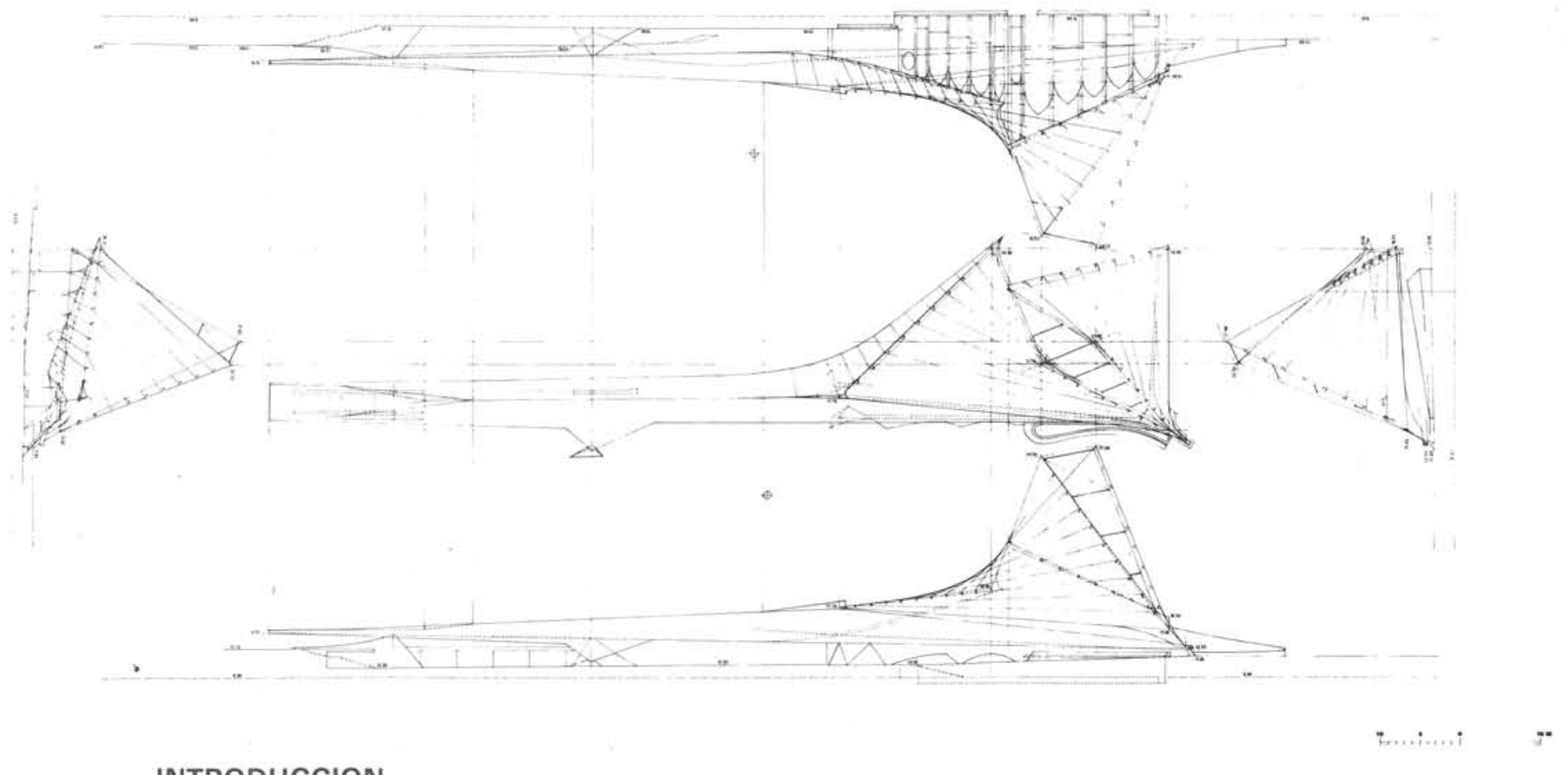

\section{INTRODUCCION}

La plaza del Viejo Mercado, lugar histórico, situado en el lado Este de la zona más animada de la ciudad normanda de Rouen forma parte del sector protegido de ésta. Este sector tiene una serie de riquezas arquitectónicas excepcionales enlazadas por calles peatonales de gran animación.

La ocasión para darle al lugar el aspecto que merecía, por su destino histórico y por sus recuerdos, vino cuando se procedió al desplazamiento del mercado de mayoristas y a la demolición de los puestos de mercado.

Los tres elementos principales: iglesia, mercado y monumento nacional, con tan distintas funciones, se han integrado en un conjunto coherente adaptado a las caracteristicas del espacio urbano que les rodea.

\section{BASES DE ACTUACION}

El acondicionamiento se realizó mediante la síntesis de varios principios complementarios:

- Unidad y simplicidad: organizar y situar en la zona los diversos elementos con objeto de crear un conjunto armonioso por su volumen, estructura y materiales.

- Diversidad y complejidad: valorar cada uno de esos elementos para que tengan un carácter propio por su forma, emplazamiento y las perspectivas ofrecidas al paseante.

- Escala humana y continuidad urbana: crear, más que un monumento, una arquitectura a la escala humana enlazando el lugar sagrado con la zona de paseo.

\section{PLANO GENERAL}

Todo el conjunto se articula alrededor del monumento nacional, realizado en el lugar donde se produjo el martirio de Juana de Arco, nexo de unión de los diferentes edificios y que sirve para el paso desde una plaza baja triangular situada al final de la calle Gros Horloge hasta una plaza-jardin, más alta, situada al norte.

Próxima al monumento nacional se encuentra la Galeria del Recuerdo, que alarga el muro de la parte alta de la plaza triangular sobre el que figuran las inscripciones que evocan el sacrificio de Juana de Arco. 


\section{Estructura}

El techo de la iglesia, que contribuye por sus prolongaciones a dar al conjunto una unidad arquitectónica, constituye un elemento dominante.

El armazón soporte de la cubierta de la iglesia, al igual que el del resto de las obras, se basa en la construcción naval tradicional que ha inspirado la realización de numerosas iglesias en Normandía, con lo que se consigue una continuidad entre el pasado y el futuro. Los diversos entramados están constituidos por unas superficies regladas, paraboloides hiperbólicos, hechas de madera y consistentes en la yuxtaposición de arcos delgados enlazados, por su plano superior, mediante otros arcos comprimidos en sentido opuesto a los anteriores.

En particular, la nave de la iglesia, que cubre una superficie de $620 \mathrm{~m}^{2}$, se realizó con dos de estas superficies alabeadas, sustentadas en su parte baja con apoyos metálicos anclados en la solera de hormigón armado y por su parte superior con un pórtico triangulado, colocado en el espacio que separa los paraboloides hiperbólicos. Este pórtico sirve, además de para dar estabilidad a la estructura, como elemento para el alumbrado de la nave.

En la nave las obras y macizos de hormigón armado se apoyan sobre un sistema de pilotes in situ, mientras que los muros inclinados de la parte sur de la iglesia, las fachadas y los pilares intermedios son sustentados por una zapata radial apoyada sobre series de pilotes verticales.

Las fachadas de la iglesia que dan a la plaza-jardín y al mercado se realizaron con pilares empotrados en el suelo, unidos entre sí por un alféizar y una solera con objeto de engarzar las trece cristaleras del siglo XVI.

\section{Materiales}

En la infraestructura y en los pilares de hormigón armado se utilizó un hormigón en tono piedra con algunas incrustaciones decorativas de ladrillos o de piedras.

En total para la realización de todas las obras de hormigón armado se necesitaron $3.500 \mathrm{~m}^{3}$ de hormigón, $150 \mathrm{t}$ de acero y $11.000 \mathrm{~m}^{2}$ de encofrado.

El entramado de la nave principal es de madera.

Para la cubierta se utilizaron placas de pizarra y, en ocasiones, de cobre.

\section{Organización del espacio}

El acceso principal de la iglesia está situado bajo el Nártex en la zona donde se unen la plaza triangular y las escaleras, muy cerca de la hoguera.

La nave principal tiene cuatro paramentos diferentes: el del sur que es un simple muro; el del este donde se abren las capillas; mientras en los otros dos están las vidrieras de la vieja iglesia de San Vicente y el acceso a la sacristía, al despacho del sacerdote y a la sala de catequesis.

El ara mayor se ha colocado en el centro de la vidriería.

Hay un coro, para un organista y una ventana de cantores, encima de la entrada principal.

Además de la entrada principal existen cuatro accesos secundarios.

\section{Las vidrieras}

La iglesia es un verdadero museo. Está cerrada por el norte con trece vidrieras del siglo XVI salvadas milagrosamente de la destrucción de la iglesia de San Vicente, en la segunda guerra mundial, por haber sido puestas en depósito anteriormente.

Para poder colocar esta magnífica tapicería de vidrio fue necesario profundizar en el suelo $2 \mathrm{~m}$ y, al mismo tiempo, utilizar de 2,50 a $3 \mathrm{~m}$ de la pendiente de la plaza. 
Esta admirable vidrieria $\left(500 \mathrm{~m}^{2}\right)$ está engarzada por una especie de emparrillado de hormigón blanco que evoca los muros de vidrio de las catedrales góticas, haciendo feliz la expresión de André Chastel: «la vidrieria aparece como factor principal de iluminación y de colorido del espacio arquitectónico.»

Las vidrieras se colocaron en marcos de aluminio.

Además de las vidrieras antiguas hay otras cristaleras de acompañamiento realizadas con una tonalidad discreta.

La protección de la vidriería antigua se realiza, teniendo en cuenta las recientes investigaciones, mediante un cristal transparente colocado en el marco de aluminio por el exterior y un poco antes de las cristaleras, con lo que se obtiene una lámina protectora de aire fácilmente renovado y filtrado.

De esta manera, a la vez que se protegen las cristaleras de los choques mecánicos y de los agentes exteriores de envejecimiento, se pueden apreciar los efectos de colorido, exteriores e interiores, creando un elemento de animación de día o de noche en este espacio público.

\section{EL NARTEX Y LA GALERIA DEL RECUERDO}

\section{EI Nártex}

Concebido como un refugio, prolongación de la iglesia, sirve de unión entre la plaza triangular y los escalones situados enfrente de la hoguera.

\section{La Galería del Recuerdo}

La galería, prolongación del Nártex y tratada de una manera idéntica, alarga el muro de la parte alta de la Plaza (Calle de Crosne). Sobre ese muro, revestido de piedra, figura una inscripción debida a André Malraux que evoca el sacrificio de Juana de Arco con estas palabras:

"Oh Juana sin sepultura y sin cara, tú que sabias que la tumba de los héroes es el corazón de los hombres.»

Esta galeria, enlosada de piedras, termina en un escalón que une por el oeste los dos niveles de la plaza. Da también acceso al pórtico de la planta baja del edificio situado al fondo de la plaza y al pequeño Museo Juana de Arco.
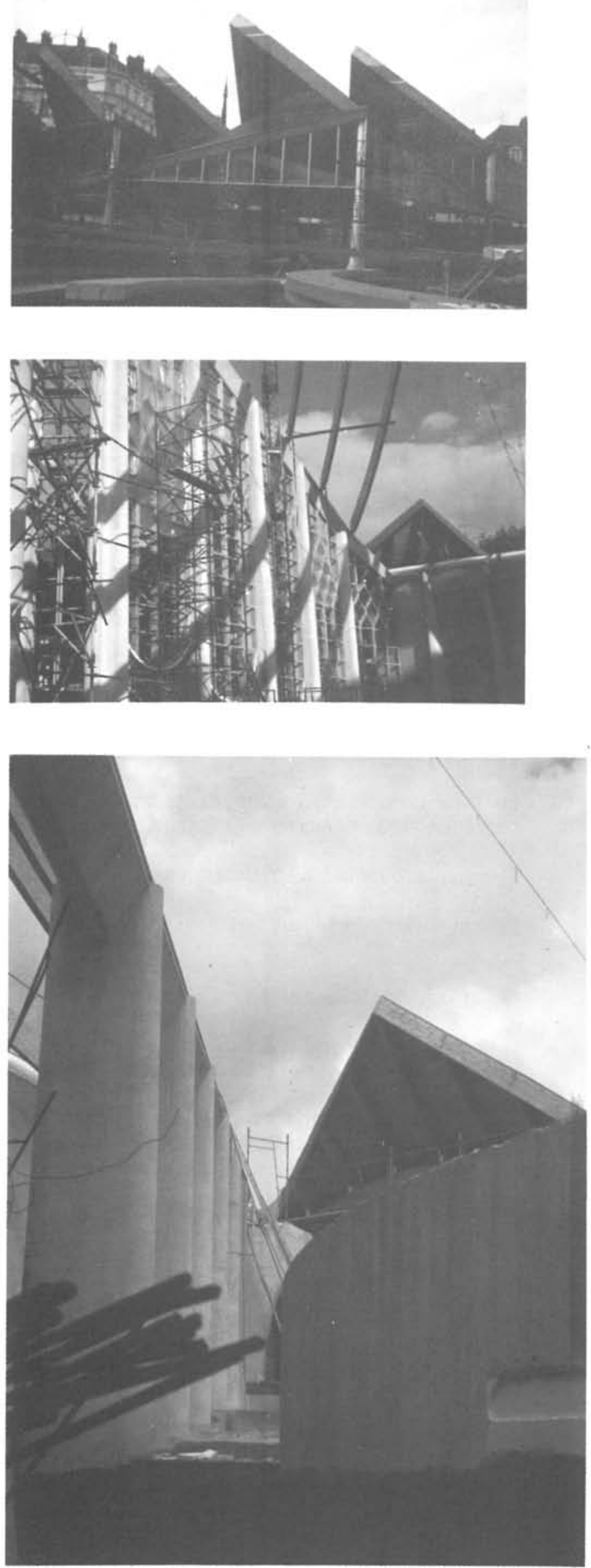

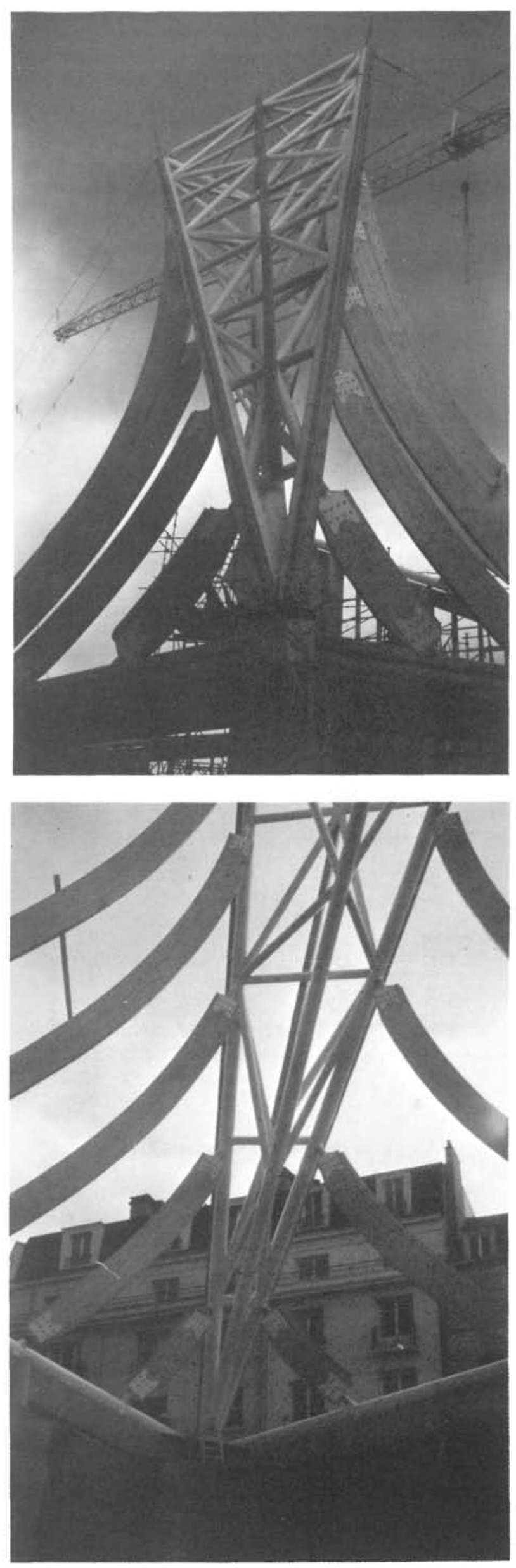

Tanto el Nártex como la Galeria del Recuerdo se han cubierto por un techo de madera, con forma de carena y revestido de placas de pizarra.

\section{EL MUSEO JUANA DE ARCO}

Al final de la Galería del Recuerdo, y al mismo nivel que ella, hay una sala de exposiciones revestida de cal para exponer las colecciones de objetos obtenidas de las excavaciones y de los descubrimientos realizados durante el acondicionamiento.

\section{EL MERCADO CUBIERTO}

Instalado al norte de la iglesia, el mercado cubierto está formado por una serie de mercadillos colocados de forma radial. Se ha hecho mediante la yuxtaposición de ocho superficies alabeadas que cubren una superficie de $1.100 \mathrm{~m}^{2}$. Los arcos se fijan en los bordes con anclajes de hierro, estando dichos bordes entrelazados por una viga de madera que hace la función de tirante para absorber los empujes principales.

Por su parte interna, el mercado hace resaltar su material de construcción: la madera; mientras que por su parte externa está revestido de placas de pizarra-tratadas en diversas tonalidades para conservar la armonia dentro del barrio, consiguiendo, aun encerrando la plaza norte, no romper la unidad arquitectónica del conjunto.

Debido a su situación el Mercado se apoya, en parte, sobre un aparcamiento cimentado a gran profundidad y, en parte, sobre el suelo natural adyacente.
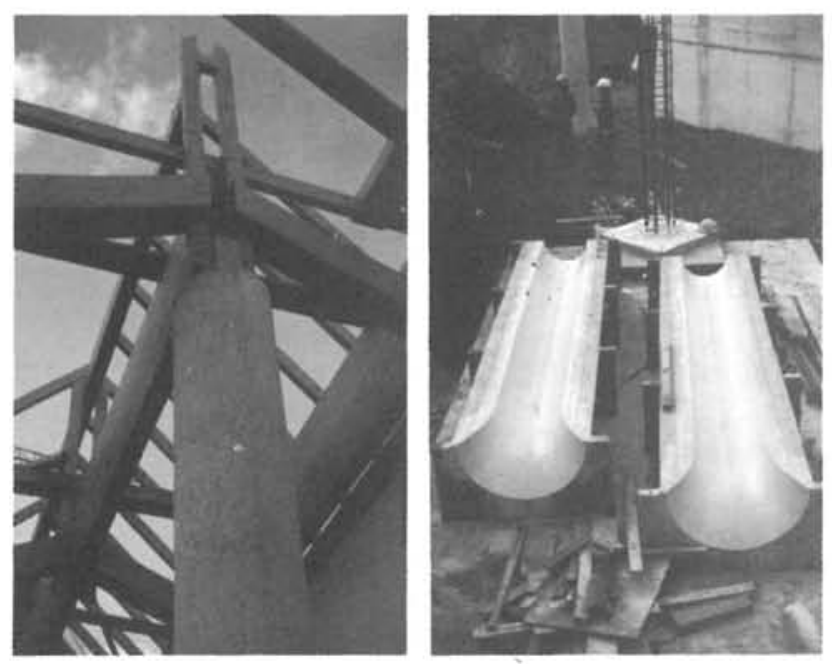
Las obras de infraestructura de hormigón armado se hacen, en la zona de suelo natural, sobre una especie de emparrillado sustentado sobre pilotes in situ, y en la del aparcamiento sobre la última planta de éste. De esta manera, la estructura se encuentra empotrada en su base y está en condiciones de transmitir a los cimientos las cargas principales de la cubierta y los esfuerzos horizontales secundarios debidos al viento.

En la superficie del mercado puede, en ocasiones, instalarse una "feria», en una especie de "calle», que une en diagonal la parte sudeste de la plaza con los escalones de la parte central que van hacia la calle Cauchoise.

\section{LA PLAZA TRIANGULAR}

A la salida de las calles de Crosne y Rollon, y en la prolongación de la calle Gros Horloge se ha hecho una plaza, que al igual que la plaza del Viejo Mercado del siglo XVI, es de forma triangular.
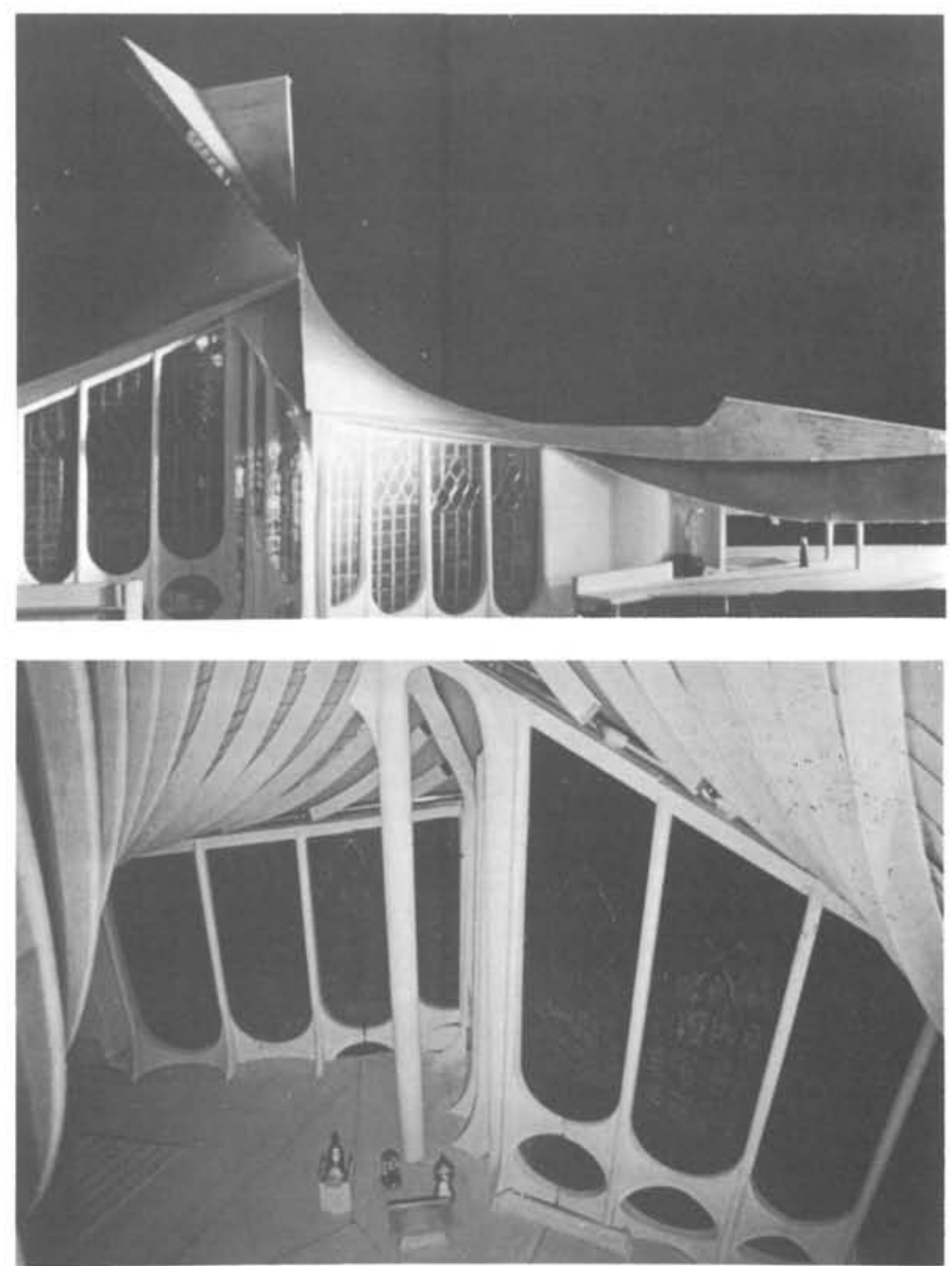

FOTOS: J.C. DE LA PANNETERIE

Esta disposición triangular realza el camino hacia la catedral por la calle Gros Horloge y crea una perspectiva cuyo efecto se encuentra reforzado por la Galería del Recuerdo, la ordenación del fondo de la plaza y el dibujo del enlosado.

El enlosado de piedra, con incrustaciones de cantos rodados, ladrillos y bandas de piedra natural sirve para exponer los restos de la iglesia de San Salvador, descubiertos en las excavaciones de 1928.

La manera de resaltar estos restos se obtuvo mediante:

- Trazado en el suelo de los muros antiguos, realizado con adoquines de colores diferentes.

- Muestra del absidiolo norte-este y del porche en el sudoeste.

- Reconstrucción en el suelo tanto de las tribunas de los jueces, como de los restos de la iglesia de San Salvador.

Al sur de la plaza, las casas antiguas de madera acentúan el carácter del espacio urbano asi creado.

\section{LA PLAZA JARDIN}

Situada al norte del Nártex y de la Galería del Recuerdo esta plaza asegura el tránsito a los peatones por todo el conjunto y ayuda a las diversas perspectivas ofrecidas al paseante.

La zona central, que forma parte del monumento a Juana de Arco, tiene unos peldaños de pendiente muy suave, junto con unas zonas de reposo, con lo que el tránsito alrededor de la hoguera, del basamento de la picota de la iglesia y del mercado está asegurado. 
El enlosado irradia alrededor de la cruz monumental destinada a perpetuar el recueruo ael sacritıcıo.

En los bordes existen plantaciones que sirven para dar vista y cerrar el conjunto.

Las vidrieras de la iglesia de San Vicente, muy visibles desde el exterior, constituyen una especie de museo al aire libre. Por la tarde, iluminando el interior de la iglesia, las vidrieras crean un espectáculo de una belleza excepcional.

\section{résumé}

AMENAGEMENT DE LA PLACE DU VIEUX MARCHE A ROUEN - FRANCE

\section{Arretche, architecte}

L'aménagement de la Place du Vieux Marché intègre, dans un ensemble harmonieux, trois èléments principaux:

-. I'église,

- le marché,

- le monument national à Jeanne d'Arc.

La structure reprend les principes utilisés dans la construction navale traditionnelle, constituee par des surfaces réglées: paraboloïdes hyperboliques en bois.

L'ensemble se distingue par les treize vitraux du XVle siécle, miraculeusement sauvés de ta destruction de l'église de Saint Vincent.

\section{summary}

CONDITIONING THE OLD MARKET SOUARE IN ROUEN - FRANCE

\section{Arretche, architect}

The aim sought in conditioning the Old Market square of Rouen has been to integrate three elements of very different func tions in a harmonious unit. These are:

the church

- the covered market, and

- the Joan of Arc national monument.

The various frameworks are based on the traditional naval construction and are made up of tuned surfaces: hyperbolic paraboloids made in wood.

The thirteen XVI century stained glass windows miraculously saved from the destruc tion of Saint Vincent's Church are an out standing feature.

\section{zusammenfassung}

UMGESTALTUNG DES ALTEN MARKPLATZES IN ROUEN - FRANKREICH

\section{Arretche, Architekt}

Mit der Umgestaltung des alten Marktplatzes von Rouen wurde das Ziel einer harmonischen Eingliederung dreier so verschiedener Elemente wie:

die Kirche.

- der gedeckte Markt und
- das Denkmal der Jungfrau von Orléans.

verfolgt.

Die verschiedenen Fachwerke gründen sich auf den herkömmlichen Schiffsbau und bestehen aus abgestimmten Flächen: hyperbolische Paraboloide aus Holz.

Im Gesamtkomplex treten die dreizehn Glasereiwerke aus dem VI. Jahrhundert hervor, die wie ein Wunder von der Zerstörung der St. Vinzenzkirche erhalten gebliegen sind. 